DOI: https://doi.org/10.46630/phm.12.2020.04

Diana M. Popović ${ }^{1}$

Article de recherche

Université de Novi Sad

УДК 821.133.1(493).09 Hellens F.

Faculté de philosophie

Reçu : le 28/2/2020

Département d'études romanes

\title{
QUELQUES RÉFLEXIONS SUR LE FANTASTIQUE RÉEL DE FRANZ HELLENS
}

L'écriture de Franz Hellens est très spécifique du point de vue d'un procédé littéraire basé sur une approche particulière de la réalité. La vision du monde de cet auteur belge est issue d'une conception que Hellens lui-même a défini comme le « fantastique réel », notion lancée auparavant par Edmond Picard à propos des Hors-le-vent, ou bien comme « les Réalités fantastiques », devenues le titre du recueil des contes de Hellens (1923). On peut constater que ce type de fantastique est une caractéristique qui domine son œuvre. L'ensemble de ses contes et nouvelles est donc cohérent avec le concept que l'auteur a par la suite théorisé, en s'appuyant sur la pensée de Picard. Nourrie de souvenirs d'enfance, du quotidien, des rêves, des oppositions clair/obscur, dehors/dedans et ainsi de suite, l'écriture de Franz Hellens bascule entre la réalité et le fantastique et se présente comme énigmatique et séduisante.

Mots-clés : contes, réalisme, fantastique, fantastique réel, littérature française de Belgique, Hellens

\section{Introduction}

Franz Hellens a laissé des traces profondes dans la littérature française de Belgique et dans la francophonie européenne. Il a été quatre fois pressenti pour le Prix Nobel $^{2}$, ce qui est un des meilleurs indicateurs de sa notoriété littéraire. Pourtant, il semble que mis à part les spécialistes, on ne connaît pas suffisamment les faits de sa vie, de son engagement littéraire ou l'environnement artistique et littéraire d'où il est issu.

Franz Hellens (1881-1972), de son vrai nom Frédéric Van Ermengem, est né à Bruxelles dans la famille d'un docteur de très grande renommée ${ }^{3}$. Il

\footnotetext{
1 diana.popovic@ff.uns.ac.rs

${ }^{2}$ En 1943, en 1948, en 1953 et en 1954.

${ }^{3}$ Son père, Émile Van Ermengem, fut professeur de bactériologie à la Faculté de Médecine de l'Université de Gand, secrétaire perpétuel de l'Académie de Médecine de Belgique, membre correspondant de l'Académie de Médecine de Paris. Il est connu pour avoir réussi à isoler la
} 
passe son enfance et sa jeunesse à Wetteren, près de Gand, et par la suite il fait ses études chez les Jésuites, au célèbre collège Sainte-Barbe ${ }^{4}$. Dans cette période il commence à écrire des poèmes et essais qu'il publie anonymement ou sous pseudonyme. La ville de Gand marquera la vie intérieure de Hellens et constituera le décor indispensable de ses œuvres littéraires. Il nourrit son écriture de la nostalgie de son enfance, notamment dans ses œuvres autobiographiques dont le personnage principal est nommé Frédéric (Le naïf, 1926 ; Les filles du désir, 1930 et Frédéric, 1935). Après avoir fini ses études de droit, en 1905, Hellens devient stagiaire à la Bibliothèque Royale (1906) et puis bibliothécaire au Parlement (1912). Il lit beaucoup et continue à écrire. Son œuvre d'avant la Grande guerre prolonge la tradition littéraire flamande de Rodenbach, Lemonnier, Eekhoud, mais elle est également inspirée par Edgar Allan Poe. Un séjour sur la Côte d'Azur et puis à Paris, durant la Première guerre mondiale, va bouleverser la vie de Hellens et va lui ouvrir un champ artistique nouveau : la peinture de Modigliani, de Matisse, d'Archipenko, le cubisme et l'art nègre, ainsi que la poésie moderne d'Apollinaire, de Cendrars et de Reverdy qui l'influenceront en tant qu'écrivain.

Mais il a su lui aussi animer les milieux artistiques. Au cours de la montée du mouvement flamand et de la flamandisation de l'université de Gand, Franz Hellens, en tant que grand bourgeois francophone de Flandre et homme d'action, crée et propage la notion de " littérature française de Belgique » dont il sera dès lors un ardent partisan et même un des chefs de file. Toute sa vie il se présente aussi comme animateur des lettres belges. Il fonde, avec André Salmon, la revue Signaux de France et de Belgique (1921), puis, avec Mélot du Dy, la revue Le Disque vert (1922-1941) ${ }^{5}$, qui accueille des écrivains tels que Blaise Cendrars, Jean Paulhan, André Malraux, Jean Cocteau, André Gide, Odilon Jean-Périer, André Baillon, Henri Michaux et ainsi de suite. En 1923 Hellens publie, dans cette dernière revue, Les réalités fantastiques, récits dont le titre pourrait s'appliquer à toute son œuvre, contemporaine et à venir. Rappelons tout de suite qu'en 1967 il va publier Le fantastique réel, un essai qui révèle, bien évidemment, que l'imaginaire hellensien naît à la jonction du réel et du surnaturel. Ces deux « qualités » sont mélangées dans une œuvre d'une forte singularité tout en faisant de Franz Hellens le chef d'un courant non réaliste, justement appelé « le fantastique réel ». Ce courant deviendra le propre de la Belgique (OTTEN 1985).

Mais on devrait dire que le goût hellensien pour ce thème n'était pas

bactérie responsable du botulisme.

${ }^{4}$ Fameux collège, fréquenté par Verhaeren, Rodenbach, Maetrlinck, Van Lerberghe...

${ }^{5}$ Un peu plus tard, il met sur pied encore deux revues littéraires, Nord (1929) et Écrits du Nord (1935). Toutes les revues que Hellens a animées entre 1920 et 1955 étaient ouvertes à toute expression de la modernité. 
isolé. Son style d'écriture a réussi à se distinguer de celui qu'on peut trouver dans la multitude d'ouvrages des écrivains belges également passionnés par le fantastique et le surnaturel. Leurs ouvrages appartiennent à d'autres courants qui surgissent au cours du vingtième siècle en Belgique francophone. Il s'agit du "symbolisme mystique » de Maeterlinck et de Rodenbach, de la « science-fiction » de J. H. Rosny, du « fantastique terrifiant » de Jean Ray, de Thomas Owen et de Gérard Prévot, du " fantastique crépusculaire » de Ghelderode, du « fantastique loufoque » de Muno, de Carême et de Quiriny, du «fantastique corrosif » de Sternberg et de Compère, ou bien du réalisme magique de Willems et de Vaes. La liste est impressionnante et comme Renata Bizek-Tatara l'a constaté à propos de ce « sujet belge », on peut dire qu'il y a si « peu de pays [qui] peuvent se vanter d'un nombre si important d'auteurs épris du surnaturel »(BIZEK-TATARA $2018: 5$ ).

Si l'on veut approcher l'œuvre de Hellens, il faudrait savoir qu'elle est immense et très variée, qu'elle comporte plus de cent volumes abordant tous les genres littéraires : contes, romans, poèmes, essais, pièces de théâtre, fables, journal intime, critiques d'art. Parmi ses écrits les plus importants, mentionnons Mélusine, fameux roman présurréaliste et onirique voué à la quête de la femme (1920 ; aux éditions Gallimard 1952), puis Moreldieu (1946), Mémoires d'Elseneur (1954). Ses Poésiescomplètes sont publiées en 1959, avant ses essais majeurs, parmi lesquels se trouvent Poétique des éléments et des mythes (1966) ou Cet âge qu'on dit grand (1970). Quant à ses mémoires, signalons l'importance particulière de ses Documents secrets, 1905-1956, parus en 1958, avec un sous-titre révélateur, Histoire sentimentale de mes livres et de quelques amitiés. On peut y trouver de très précieuses informations sur sa vie et son art d'écrire.

Même si entre 1947 et 1971 Hellens séjourne en France, il songe toujours à son pays d'enfance. Finalement il rentre dans sa ville natale, en 1971, où il décède un an plus tard, à l'âge de 90 ans. Mais plus d'une décennie auparavant, il a déjà résumé sa vie et également son écriture dans ces mots, qu'il a gravés dans ses Documents secrets (HELLENS 1958 : 50) :

«C'est toujours à mon pays d'origine que sont allées mes pensées les meilleures. Et particulièrement à Gand et à cette région de Flandre baignée par la Lys et l'Escaut, où s'est écoulée mon enfance et la plus grande partie de ma jeunesse. J'y reste attaché par toutes mes fibres. Seul l'amour de la langue que je parle et que j'écris, et qui fut celle de mon berceau, m'a attiré dans le pays où elle est le mieux parlée et le plus correctement écrite. »

Cet attachement à son pays et à sa langue est omniprésent dans ses écrits. Son retour aux sources est en effet un retour vers un passé privilégié, à son enfance. Mais parmi ses thèmes majeurs, outre l'enfance, on devrait de même mentionner la femme aimée mais insaisissable, le rêve, l'énigme du mal, et 
surtout la métamorphose du réel. Ce dernier nous intéresse particulièrement et nous y consacrerons les lignes qui suivent, puisqu'elle constitue l'un des traits constants de l'écriture hellensienne et de son œuvre littéraire.

\section{Autour des notions « réalisme fantastique » et « fantastique réel »}

À partir des années vingt, Franz Hellens a tâché de développer une conception appelée « le réalisme fantastique » ou bien « le fantastique réel ». Pour lui, il ne s'agissait pas d'une simple formule, mais plutôt d'une attitude, une manière de voir, de sentir ou d'imaginer les choses (HELLENS 1967 : 9). En effet, son « réalisme fantastique », c'est son rapport actif à la quotidienneté et en quelque sorte un « instrument d'investigation psychologique et d'expression littéraire » (BIZEK-TATARA $2018: 6$ ). Mais il ne consent jamais à définir cette notion, puisqu'il est d'avis que les mots ne suffisent pas pour le faire : atteindre aux sources du fantastique réel se fait, d'après lui, autrement et non «par le truchement des mots du vocabulaire» (HELLENS 1967 : 53). Mais, avant d'aborder encore plus en détails son point de vue, expliquons comment ce terme à été lancé dans la littérature.

Parmi les critiques contemporains, la notion de «fantastique réel » a souvent été liée à celle de " réalismemagique ». Mais, d'après Weisgerber (1987 : 301), ce dernier n'a été utilisé qu'à partir de 1925 et qui plus est hors de la Belgique, en Allemagne. Cela veut dire que la notion de « réalisme magique » vient beaucoup plus tardivement que celle de " fantastiqueréel », déjà connue en Belgique à la fin du dix-neuvième siècle. En 1910 Edmond Picard a, avec raison, appliqué, le terme «fantastique réel» à Hellens. Rappelons qu'il a décrit l'atmosphère spécifique du recueil hellensien de contes intitulé Hors-le-Vent, paru en 1909, raison pour laquelle cette œuvre a été dès lors considérée comme la première de ce genre (ce qui est faux, on va bientôt l'expliquer). Par la suite, Hellens va emprunter cette notion à Picard et la théoriser.

En poursuivant ces recherches, on s'aperçoit qu'Edmond Picard avait, en effet, proposé cette notion plus de deux décennies avant de l'utiliser à propos de l'œuvre de Hellens : c'était en 1887, pour présenter deux de ses propres œuvres, La Veillée de l'huissier, un conte de Noël, paru en 1885, et LeJuré, un monodrame en cinq actes, paru en 1887. D'après Éric Lysøe, Picard a emprunté ce syntagme oxymorique à un critique français, Jules Claretie, en l'employant d'une manière différente de Hellens. Lysøe constate que Picard comprend le « fantastique réel» comme une notion où s'unissent les caractères « naturaliste » et « symboliste ». Par la suite, le critique explique cette idée en 
s'appuyant sur des faits socio-culturels, qu'il voit comme « binaires », et qui forment ce caractère « belge » d'un écrivain (LYSØE 1994 : 100) :

« Par son caractère binaire, la formule correspond assez bien à la représentation duale que l'on commence à donner de la Belgique. "Fantastique" s'élargit en rêve, en mysticisme, "réel" renvoie au réalisme, au souci du détail observé par un œil de peintre, et l'ensemble se confond ainsi avec les deux registres qui servent à définir l'“âme belge" ".

Et quand Picard écrit à propos des Hors-le-Vent, il ne se réfère qu'au « symbolisme ». On pourrait associer ce recueil à L'Intruse de Maeterlinck ou aux ouvrages de Van Lerberghe, et donc de manière erronée au « réalisme magique ». Lysøe dans son analyse conclut que " comme on peut voir, cela n'a rien à voir avec le réalisme magique, mais plutôt d'un fait identitaire " (LYSØE 1994 : 86). En réalité, l'histoire littéraire a bien attesté que le " fantastique réel » est resté un fait « belge ». Et lorsque Hellens utilise ce terme qu'il a repris à Picard et le terme « réalisme fantastique », il le fait pour désigner une seule direction d'écriture, celle qui le détachera de l'héritage symboliste. En effet, il cherchait à se distinguer de ses prédécesseurs et à créer sa voie (sa voix) propre.

\section{La conception hellensienne du « fantastique réel »}

Trois ans avant les Hors-le-Vent Hellens a publié un roman, En ville morte (1906), dont le titre même révèle que l'écrivain a subi les influences symbolistes. On peut dire que le recueil Hors-le-Vent est aussi lié à ce mouvement, surtout thématiquement, mais il faudrait souligner que cela n'est le cas dans aucun des six textes dont le recueil est composé. Cela marque déjà un signe d'une certaine rupture avec l'héritage symboliste. Si Hellens est féru des idées picardiennes (et par la suite reprend sa terminologie), ce n'est pas pour les suivre aveuglément, mais pour prouver que le « fantastique réel » pourrait être interprété un peu différemment.

Rappelons que Picard a parlé du " mystère » qui se cache derrière l'apparence du réel, un " mystère » que « les yeux vulgaires » ne sont pas capables de voir (PICARD 1887 : 35). C'est donc le regard qui est dans ce cas d'une importance considérable, puisque le fantastique est en relation directe avec un regard et une interprétation particulière de l'écrivain, ce qui veut dire que le fantastique ne réside pas dans l'objet, mais dans l'œil. D'après Picard, le fantastique s'appuie sur la réalité, il est même ancré dans la réalité, et ce qui nous reste à faire c'est le saisir. Autrement dit, il faut savoir regarder la réalité et par la suite avoir la capacité de percevoir ce qui est celé au regard et également de pénétrer un « ailleurs », pour entrevoir une autre face du visible 
(BRION 1957 : 137). On peut constater que c'est un don, un privilège de l'écrivain.

Picard insiste sur l'idée qu'il s'agit de l'interprétation particulière de celui qui regarde. Hellens s'approprie cette idée et fait un pas de plus. Pour lui, le réel et le surnaturel sont intimement liés. Il dit que le fantastique «n'abandonne jamais la réalité, il l'élargit »(HELLENS 1958 : 7). De plus, il est d'avis que le fantastique fait partie intégrante de la réalité. C'est justement ce « mystère » picardien - mentionné plus haut - bien caché mais présent dans le réel. Pour Hellens, le rôle qu'il attribue à l'écrivain, c'est de faire surgir cet élément mystérieux du réel :

«S'avancer tellement dans le frisson du concret que l'imaginaire éclate comme de lui-même de la réalité élue, tel est le cheminement le plus secret mais le plus efficace d'une certaine conception tout irrationnelle de l'esprit et de la sensibilité. » (HELLENS 1967 : 17).

Mais, pour Hellens, le fantastique réel est lié à la vie de tous les jours et à un entourage familier. Il ne le lie jamais aux êtres de l'au-delà, par exemple aux fantômes, à Dieu ou au diable, ni aux êtres maléfiques, terrifiants ou odieux. Et en plus, chez lui, comme Picard l'a commentéà propos des Hors-leVent, il s'agit d'un soupir, d'un glissement, d'une vibration, d'un craquement qui modifie les choses, sans qu'il y ait eu une modification. En fait, la réalité demeure à la fois même et " autre " (PICARD 1910 : 1). Ce qui incite le surgissement du fantastique réel, ce ne sont pas des phénomènes de l'au-delà ou surnaturels, mais c'est une approche au réel et un certain regard, « un regard plus vrai sur la réalité concrète, regard révélateur l'entrevoyant infiniment plus riche, plus complexe et foisonnante qu'une perception ordinaire, lissée par l'habitude, ne perçoit pas » (BIZEK-TATARA 2018 : 11). Il s'agit donc d'un état d'esprit et de la conscience du personnage, et non pas d'un agent surnaturel. C'est justement ce qui distingue le fantastique réel du fantastique. Ce « regard plus vrai » est donc nécessaire pour mieux comprendre la réalité (et le monde en général), c'est pourquoi on peut dire que le fantastique réel a une valeur révélatrice et cognitive. La connaissance du monde s'élargit et on entre dans un domaine « dépaysé » de la réalité (HELLENS 1992 : 103), dans cet autre côté du miroir. Par exemple, la fumée des cigares peut modifier le regard du personnage, comme dans le récit La Cuisine des fous, le plus long d'entre les récits des Hors-le-Vent, où le cigare prend pratiquement une place centrale (HELLENS $1912: 20$ ) :

«Rien n'était étrange comme la fumée qui s'étirait dans cette chambre étriquée. [...] Peu à peu, son nuage léger de petites cendres piquantes descendait vers nous, et, par tous les pores, glissait dans nos cerveaux des images de folie et de rêves. » 
Par la suite on s'aperçoit que la fumée du cigare stimule l'imaginaire. La chambre, d'abord décrite d'une manière réaliste comme étroite et malsaine, devient par la suite le lieu fantasmatique par excellence. C'est la présence de la fumée qui a provoqué ce changement. Elle modifie le regard du narrateur et dirige un dédoublement du point de vue narratif.

Pour mieux éclairer les choses, Hellens se sert aussi des contrastes, dans un sens thématique ou spatial. Dans ce jeu des oppositions il trouve la possibilité de montrer le passage du réel au fantastique et vice versa. Dans le récit $A u$ repos de la santé, on voit un passage de la fièvre et la maladie, à travers le repos et le rêve vers la santé. L'opposition maladie/santé se complète d'autres oppositions signifiantes (ville/auberge, jour/nuit, réel/rêve, danger/ sécurité, absence/présence et ainsi de suite). On voit aussi une distinction entre ce qui est dehors et ce qui est dedans. Cela est encore plus prononcé dans le récit Entre la rue et le jardin où la place privilégiée est une classe. Le glissement vers le fantastique est relié aux trois positions (classe - rue jardin) où le narrateur rappelle les souvenirs de son enfance (HALEN 1985 : 47-59). Les deux perspectives, de près et de loin, se complètent et contribuent à l'unité du récit.

Dans la conception du fantastique réel hellensien, le rêve prend une part importante. Il permet de saisir une vérité supérieure, même de vivre et d'éprouver les désirs les plus profonds. Il possède des vertus révélatrices, puisque le rêve, éveillé ou non, se comporte comme un écran qui montre un monde subjectif qui fait en même temps indéniablement partie intégrante de notre existence objective. Hellens est fasciné et dit : «Quoi de plus réel, de plus vrai, de plus détaché des contingences raisonneuses, que le rêve, cauchemar ou songe bienheureux !» (HELLENS 1967 : 68). Il est d'avis que le rêve est comme une porte ouverte vers l'infini. L'écrivain le représente comme vraisemblable, très vif, très suggestif, il se mêle à la vie et très souvent le lecteur ne peut plus distinguer le moment où l'on passe d'un monde à l'autre, de la vision onirique à la réalité et vice versa. Cela permet de saisir plus profondément un même fait du récit. Ce procédé se rapproche de l'idée d'une constante hésitation, mentionnée par Tzvetan Todorov dans L'introduction à la littérature fantastique : " Le fantastique, c'est l'hésitation éprouvée par un être qui ne connait que les lois naturelles, face à un événement en apparence surnaturel » (TODOROV 1976 : 29). Cette hésitation, en tant que procédé littéraire, se présente comme un champ également prolifique pour la création hellensienne. Dans son œuvre on peut remarquer qu'il utilise très souvent les motifs oniriques et une " écriture d'indécision » qui multiplient les aspects des choses et des êtres.

Encore plus que le rêve, éveillé ou non, il existe quelque chose qui s'imprime beaucoup plus dans la mémoire et donne un accès à la connaissance de la quotidienneté : le hasard. Cela met à distance l'objet pour mieux le voir et 
le comprendre. D'après Hellens, il s'agit d'un moment miraculeux et insolite, qui nous rend muets et instruit (HELLENS 1966:67) :

« Le hasard est la forme la plus sensible de l'univers, la plus efficace aussi, puisqu'elle étonne et par là s'imprime le mieux dans la mémoire. Il est son miracle permanent. [...] Il fait la clarté sur tout, sans besoin d'explication. Il pose le problème et en même temps le résout. "

Il est évident que chez Hellens cette orientation vers l'étrange (le réalisme onirique, le hasard) ne fait jamais l'opposition entre fantastique et réel, mais crée une image complexe qui suscite un sens d'une parfaite complémentarité entre ces deux natures.

Il faudrait aussi mentionner que le fantastique réel chez Hellens est dépourvu d'émotions fortement négatives. " Le fantastique réel n'est pas nécessairement générateur d'épouvante, mais il n'exclut ni le tremblement ni l'angoisse », dit-il (HELLENS 1967 : 61) et continue avec l'idée que pour obtenir un effet de trouble, " il n'est pas indispensable de faire appel à des monstres, si beau soient-ils, infernaux ou terrestres » (HELLENS 1967 : 61). Lorsqu'il présente les émotions, ce sont très souvent de différentes manifestations de malaise, une inquiétude, une angoisse, un dérangement ou une peur diffuses et donnent une expérience particulière d'un sentiment étrange. Mais le personnage éprouve ce sentiment d'une manière tout à fait ambiguë, puisque ce sentiment le dérange et le trouble et en même temps il l'attire en lui procurant une certaine jouissance. Parfois il est obsédé par cette énigme et il cherche à raviver une même expérience, même si elle est inquiétante. C'est comme dans le récit $L a$ courge où le narrateur dit : «Depuis, je ne passai aucun jour pour éprouver le désir intense de retrouver cette inquiétante hallucination. » (HELLENS 1967 : 16). Ce double rapport est encore une fois une illustration de l'idée hellensienne que toute chose dépend de notre regard et de notre approche de la réalité, ce qui stimule notre imagination et enrichit notre esprit.

\section{En guise de conclusion}

L'écriture de Franz Hellens repose sur un procédé qui a une tonalité symboliste, mais elle est dans le même temps ancrée dans le réalisme. L'auteur crée une atmosphère suggestive et ne cherche pas à convaincre le lecteur ni à expliquer en détail que l'intrusion du surnaturel a vraiment eu lieu. Il cherche, tout simplement, à présenter une réalité. Mais cette réalité est plus vaste que ce que la perception de nos sens physiques sous-entend : on peut y trouver mêlés des traits qui élargissent le réel. Picard et Hellens le comprenaient comme une sorte de mystère qui fait partie intégrante du réel. C'est ce qui demeure dans leur esthétique du fantastique réel, tout en le détachant du fantastique 
dit classique. Chez Hellens le mystère est une donnée inhérente à la réalité, par conséquent il existe une relation de réciprocité entre les deux parties de la notion « fantastique réel » : c'est substantif qualifié par un adjectif et adjectif qualifiant un substantif. Cela veut dire que le réel est étrange et mystérieux, autrement dit fantastique, et que le fantastique vu comme l'étrange et l'onirique, l'insolite ou le magique est une donnée réelle. Son écriture oscille donc incessamment entre une expression réaliste et une autre, fantasmatique.

Le fantastique réel hellensien est lié au quotidien et ne dépend que de l'approche personnelle et particulière du protagoniste. Il s'agit d'une question d'optique du narrateur et de sa propre expérience et non pas d'une intervention d'un être ou d'un événement surnaturel ou méconnu. Tout provient d'un monde familier, proche, contemporain ou révolu. S'il existe un dédoublement du point de vue narratif, c'est que la narration est régie par un certain stimulant qui modifie le regard du narrateur et provoque son imaginaire en lui donnant accès à une réalité « élargie », une réalité qui se cache sous les apparences. C'est là sans doute ce qui attire le narrateur. De plus, il faudrait constater que même si le passage à cet autre monde est très souvent suivi d'un sentiment insolite ou inquiétant, il se présente comme une énigme qui séduit le narrateur et le lecteur.

$\mathrm{Au}$ terme de cette rapide analyse on doit constater que les œuvres hellensiennes ont réussi à constituer une certaine « école du regard » qui ont inspiré toute une génération d'écrivains fantastiques belges. Franz Hellens a proposé une esthétique de la divagation et a pris une place emblématique dans l'histoire littéraire, surtout en Belgique francophone.

\section{Bibliographie}

BIZEK-TATARA 2018 : BIZEK-TATARA, Renata. « Le fantastique réel de Frantz Hellens. Genèse et conception ». Écho des études romanes $\mathrm{n}^{\circ} 1-2$ (2018) : 5-14.

BRION 1957 : BRION, Jean-Baptiste. « Le fantastique dans l'œuvre de Franz Hellens ». Hommage à Franz Hellens. Paris : Albin Michel, 1957 : 131-139.

HALEN 1985 : HALEN, Pierre. «Un certain regard sur le monde. Le fantastique réel dans l'œuvre de Franz Hellens ». Otten, Michel (dir.). Écritures de l'imaginaire. Bruxelles : Labor, 1985 : 45-67.

HELLENS 1958 : HELLENS, Franz. Documents secrets (1905-1959). Hitoire sentimentale de mes livres et de quelques amitiés. Paris : Albin Michel, 1958.

HELLENS 1967 : HELLENS, Franz. Le fantastique réel. Bruxelles - Amiens : Sodi, 1967.

HELLENS 1912 : HELLENS, Franz. Les Hors-le-Vent. Bruxelles : La librairie moderne, 1912. 
HELLENS 1966 : HELLENS, Franz. Poétique des éléments et des mythes. Paris : Albin Michel, 1966.

HELLENS 1992 : HELLENS, Franz. Retour du fantastique. Bruxelles : Labor, 1992.

LYSØE 1994 : LYSØE, Éric. «Franz Hellens et le fantastique réel : quelques jalons pour l'arpenteur ». La littérature belge de langue française. Initéraires et contacts de cultures $\mathrm{n}^{\circ} 20$ (1994) : 85-101.

OTTEN 1985 : OTTEN, Michel. «Franz Hellens et le réalisme fantastique ». Le fantastique dans les lettres françaises de Belgique. Cahiers du CERCLEF no 3 (1985) : 45-53.

PICARD 1910 : PICARD, Edmond. « Franz Hellens. Le fantastique reel ». La Chronique 18. 12. $1910: 1-3$.

PICARD 1887 : PICARD, Edmond. Le Juré. Bruxelles : Des presses de Mme $V^{\mathrm{e}}$ Monnom, 1887.

TODOROV 1976 : TODOROV, Tzvetan. Introduction à la littérature fantastique. Paris : Éditions du Seuil, 1976.

WEISGERBER 1987 : WEISGERBER, Jean (dir.). Le Réalisme magique. Lausanne : L'Âge d'homme, 1987.

Диана М. Поповић

\section{НЕКА РАЗМАТРАЮА О ФАНТАСТИЧНОМ РЕАЛИЗМУ ФРАНЦА ХЕЛЕНСА}

Начин на који Франц Хеленс пише врло је специфичан. Његова визија света темељи се на концепцији коју је он са̂м дефинисао као „реална фантастика" - синтагмом насталој према појму који је увео Едмон Пикар описујући Хеленсову збирку Затишје - или као „фантастична стварност” - синтагмом коју ће нешто касније Хеленс искористити да наслови једну своју збирку прича (1923). Можемо видети да је фантастично постало доминантна одлика читавог књижевног стваралаштва овог белгијског аутора. Све његове приче и новеле доследне су том концепту, који ће он и теоријски појаснити, ослањајући се, између осталог, на Пикара. Хеленсово прозно стваралаштво базира се на ауторовим личним сећањима из доба детињства, на свакодневици, на сновима, на супротстављеним појмовима као што су светло/тама, споља/изнутра и тако редом, и вешто осцилује између света стварности и фантастике, одајући утисак нечег врло загонетног, а у исти мах привлачног.

Кључне речи: приповетке, реализам, фантастично, фантастични реализам, белгијска француска књижевност, Хеленс 